\title{
Intraorganelle Nanoporation in Biomedical Application
}

\author{
S.Sarkar \\ ECE, SMIT, Sikkim, India
}

\author{
M.K.Ghose \\ Dean Academics,SMIT,India
}

\begin{abstract}
Intraorganlle nanopoation is a highly effective method to increase permeability of intraorganelle membrane under the influences of pico electric pulses and using this technique, we can introduce specific drugs into the intraorganelle of the regid cell like osteoblast. It is also a promising technology in drug delivery system and localization of malignant cancer cell. Till now it is reported that the reversal electroporation suffers from an inability to destroy large volumes of cancer tissue without introduction of cytotoxic elements and increasing the applied electrical field to the harm full level. This restriction can be overcome by using intraorganelle nanoporation which is described here. In this paper we numerically model a micro device used for characterization of osteo intraorganelle nanoporation and different field of biomedical application
\end{abstract}

\section{Keywords}

Intraorganele nanoporation, pulse electric field, micro device, membrane pore, biomedical application

\section{INTRODUCTION}

The effect of intense pulsed electric fields on biological cells and tissues has been reported since the late 1950's. The role of short and intense electric fields on biological cells have been studied for a long time as they are known to apply interrupt on the plasma membrane which can become permeable to various molecules [1].This temporary permeabilization of the plasma membrane allows genes or drugs entering into the cell intraorganelle. This technique is very attractive in various applications such as electrochemotherapy [2][3], cutaneous and subcutaneous tumour nodule treatments [4],[5], evaluation of the cytotoxicity of nonpermeant or poorly permeant anticancer drugs [6], gene electrotransfer to various animal tissues [8] and latterly with in various tests [7].Different works have reported about the effects on animal cells of these kinds of pulses, commonly named nanosecond pulsed electric fields (nsPEF). More precisely, it has been shown that the gene electrotransfection efficiency can be improved by nsPEF exposure, also called nanoporation, [12] and that disturbances on the cell membrane could be sufficient to render it permeable to small molecules, such as propidium iodide [10],[11]. Beyond their potential effects on the cell membrane, these nsPEF show a great interest because they also offer the possibility to disturb the intra cellular structures and functions [13]. However, the mechanisms implied in the effects of psPEF on living cells remain still misunderstood. This is mainly due to the lack of real-time visualization and monitoring systems during psPEF application on biological cells.

Permeabilization of the cell plasmamembranewith highamplitude electric pulses has been known for decades and is commonly employed for intracellular delivery of xenobiotics and cell destruction [14-17]. Shortening the pulse duration into nanosecond range has the potential

to permeabilize not only the plasma membrane, but the intracellular membranous structures as well [18-21]. Multiple studies have attempted to verify this theoretical prediction, with rather diverse outcomes. Depending on the experimental approach, the studies reported only plasma membrane effects [22-26].

In the other hand Electric stimulation has been used successfully to treat a wide range of bone disorders. However, the mechanism by which the electric fields can influence the bone cells behavior remains poorly understood. The purpose of this research was to assess the possible mechanism of the stimulatory effect of pulsed electromagnetic field (PEMF) and intraorganelle pore formation on osteoblast like bone bone cells. Electric fields have been well established to modulate bone physiology [27]-[30]. However, despite a multitude of studies of bone-derived cell cultures with electric fields [31][34], little is known about the mechanism exerted by the cells to sense an electric field, how they convert the information into a biochemical signal, and generate the appropriate physiological response. Therefore further research is required to determine this interaction process between electric fields and bone cells. This was exactly the objective that motivated the present work "to identify the possible pathway(s) of interaction between electric fields and bone cells". Establishment of the exact nature of electric field transduction would not only solve a fundamental mystery in the field of bone research, but would provide valuable benefits in the management of bone diseases with electrical therapy. However, despite an abundance of undeniably valid data, from both basic and multicenter clinical trials, demonstrating the effects of electrical stimulation on skeletal biology and healing, little is known about the signal transduction that mediates the physiological response of bone to electrical stimulation. As consequence, a certain amount of skepticism remains among biologists and clinicians, due to the fact sometimes the method used is not the appropriate one and can thus bring collateral damages. Therefore scientists need to learn more about how electricity interacts with bone, thereby it will provide more effective electrical stimulators and prevent principally secondary damages. Until recently, it was only believed that such alterations in the electrical environment of bone are perceived by bone cells which in turn initiate a chain of intracellular events that lead to an adaptive bone formation. However it has not been proved to date. To try to solve such a dilemma, a multitude of in vitro studies involving bone cells and electrical stimulation have been performed over the last 20 years. As electrical stimulation has been used electric and magnetic fields, direct current and ultrasound among others [31]-[34]. Bone cells have responded in different manners that are considered key in the process of bone formation. Therefore it has not been questionable if bone cells respond to electrical stimulation, but what is the mechanism that is acting on the cells, and how it is transduced into biochemical signals for generating an appropriate physiological response. In light of all research done with bone and electricity, there is enough evidence to say that electric fields exist into bone and play a role in bone physiology, and that external electrical stimulation can also alter profoundly many cellular events and modulate the bone physiology but the mechanism for pore formation is not properly investigate.However, until the advance mechanism of interaction between electric fields and bone cells placed in a microfloudics is limited, little advance can be made in the 
intraorganelle pore formation into the osteoblast like bone cell for the management of bone diseases with electrotherapy.

Nanoporation-based technologies and medical applications have already shown their laboratory and clinical relevance. It is becoming a standard appliances in bi-medical applications are in progress. Although the number of successful applications is increasing, several questions concerning the optimization of pulse parameters for specific application are still open. Among them is determination of appropriate amplitude, duration, number, and repetition frequency of electric pulses that assure successful application or treatment with minimal possible side effects.

Traditional nano-poration is done in batches of cells between electrodes and there is little control over the permeabilization of individual cells. Therefore, it is very difficult to study the fundamental biophysics of cell membrane electropermeabilization and to design optimal and reversible electroporation protocols for individual cells [3536]. Although the biophysics of poration are still not fully understood, indirect evidence shows that micro aqueous pores with diameters of tens to hundreds of Angstroms eletrical field induced structural rearrangement of the lipid bilayer [37]. It occurred to us that if nanoporation induces pores in the lipid membrane, then, in a state of poration, a measurable current should low through individual cells, which otherwise are impermeable to electrical currents. Based on this idea, we have developed a new microelectroporation technology to study and control the nanoporation process of individual cells. The microelectroporation chip was designed and fabricated using standard silicon micro fabrication technology. With the ability to manipulate individual cells, the chip can be used to study the fundamental biophysics of intraorganelle nanoporation of single osteoblast like-cell level and in biotechnology, for controlled introduction of macromolecules, such as gene constructs, into individual cells. The general principle of the microelectroporation technology and the design, fabrication of chip and the characterization of nanoporations are presented After the first description of in vitro gene transfer to living cells by intraorganele nanoporation (EP) this technique has been widely used. The use of intraorganele nanoporation to transport non-permeant chemotherapy drugs has been termed electrchemotherapy.

In this paper major focus will be on mathematical, analytical of nanoporation and simulation model of 3D micro device used for intraorganele characterization for osteoblast cell and various bio-medical applications under the influences of pico electric field and emphasis is to analyze interactions in between pulse electric field with intraorganelle materials, including membranes and other cellular components for biomedical application.

\section{THEORETICAL MODELS}

\subsection{Mathematical modelling of nanoporation}

The Intraorganelle nanoporation process, including the formation and expansion of the pores, are described by the Smoluchowski [36] partial differential equation. The Smoluchowski equation defines a pore density function, $n(r$, $t$ ), such that the number of pores with radius between $r$ and $r+$ $d r$ at any given time, $t$, is $n(r, t) d r . n(r, t)$ is described as $\frac{\partial n}{\partial t}+D \frac{\partial}{\partial r}\left(-\frac{\partial n}{\partial r}-\frac{n}{k T} \frac{\partial W}{\partial r}\right)=S(r)----------(1)$

Where $D$ is the pore diffusion coefficient, $r$ is the pore radius, $W$ is the formation energy of a pore with radius $r$, and $S(r)$ describes the transition of hydrophobic pores to hydrophilic ones, as

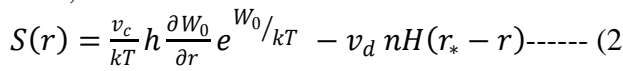

where $v c$ is the pore creation rate, $h$ is the membrane thickness, Wo is the formation energy of a hydrophobic pore, $v d$ is the pore destruction rate, $r *$ is the radius at which hydrophobic and hydrophilic pores have the same energy, and $H(r *-r)$ is a step function at $r=r *$.Assuming that, $(i)$ the expansion of the pores is negligible, and (ii) the temporal change of the minimum pore energy is negligible, a quasistatic asymptotic model of Intraorganelle nanoporation simplifies the PDE equation to an ordinary differential equation. The ODE defines the pore density, $N(t)$, which is related to $n(r, t)$ as

$N(t)=\int_{r=0}^{\infty} n(r, t) d r$

The quasistatic asymptotic equation for $N(t)$ is $\frac{d N(t)}{d t}=\propto e^{\left(V_{m}(t) / V_{e p}\right)^{2}}\left(1-\frac{N(t)}{N_{0}} e^{-q\left(V_{m}(t) / V_{e p}\right)^{2}}\right)-$ (4)

Where $V m$ is the transmembrane voltage, Vep is the characteristic voltage of Intraorganelle nanoporation, $N O$ is the equilibrium pore density at $V m=0$, and $a$ and $q$ are constants.

It is reported that,when the transmembrane voltage achieves the required voltage of Intraorganelle nanoporation some pores are formed in the membrane. The formation of the pores increases the membrane conductivity of the membrane and is electrically modelled as an additional current density, $J e p$, inside the membrane. Jep is written as

$J_{e p}(t)=N(t) \frac{\pi r_{p}{ }^{2}}{h} \sigma_{p} V_{m} K$

where $N$ is the density of the pores, $r p$ is the pore radius, $\sigma p$ is the conductivity of the solution inside the pore, $V m$ is the transmembrane voltage, $h$ is the thickness of the membrane, and $K$ is

$K=\frac{e^{v_{m}}-1}{\frac{\omega_{0} e^{\omega} 0-n v_{m}-n v_{m}}{\omega_{0}-n v_{m}} e_{m}^{v}-\frac{\omega_{0} e^{\omega} 0+n v_{m}+n v_{m}}{\omega_{0}+n v_{m}}}-$

Where $w 0$ is the energy barrier inside the pore, $n$ is the relative entrance length of the pore, and $v m=q e, k T V m$ is the non-dimensional transmembrane voltage. Assuming that the electric field inside the membrane is uniform, the transmembrane voltage is written as

$V_{m}=E_{*} h$

Where $E_{*}$ is normal electric field.

The pore current density inside the membrane can be translated as an increase in the membrane conductivity using the relation

$J_{c p}=\sigma_{m} E_{*}$

The conductivity of the membrane at the points that pores are formed is calculated as

$\sigma_{m}(t)=\sigma_{m 0}+\mathrm{N}(\mathrm{t}) \sigma_{\mathrm{p}} \pi \mathrm{r}_{\mathrm{p}}{ }^{2} \mathrm{~K}$

where $\sigma m 0$ is the conductivity of the membrane before Intraorganelle nanoporation. The nonlinearity of the equation 3.4 comes from $N$ which was described as $\frac{d N(t)}{d t}=\propto e^{\left(V_{m}(t) / V_{e p}\right)^{2}}\left(1-\frac{N(t)}{N_{0}} e^{-q\left(V_{m}(t) / V_{e p}\right)^{2}}\right)-(10)$

Under equilibrium conditions (9) and (10) maintain the transmembrane voltage below the required voltage of Intraorganelle nanoporation, which is about $1 \mathrm{~V}$.Required voltage of nanoporation is the threshold at which notable increase in the density of the pores and membrane conductivity and consequently decrease in the transmembrane voltage occur. The increase in the transmembrane voltage leads to the increase of pore density which lowers the conductivity of the membrane and subsequently the transmembrane voltage.

\subsection{Analytical model of nanoporation}

We will initially focus on a simple analytical, passive, and linear model of the cell. However, it provides useful information on the threshold for the onset of nonlinear effects. 
After introducing the concept of electro-effects that depend on pulse duration, we will discuss advanced models that include changes in cell structures.

The assumptions used in most models for membrane charging and Intraorganelle nanoporation as well as intracellular electro-manipulation are that the membranes are perfect insulators, and that the permittivity of the liquids in and outside the cell can always be neglected. Starting from the resting voltage, $V_{r}$, (which is on the order of $70 \mathrm{mV}$ for many cells), the voltage across the plasma membrane will increase with time until the end of the pulse at time $\tau$, and reach a value of $V_{n}(\tau)$ at the poles given by

$V_{n}(\tau)=f E(D / 2)\left[1-\exp ^{\left(-\tau / \tau_{c}\right)}\right]$

Where $f$ is a geometry factor ( 1.5 for spherical cells), and D is the diameter of the (spherical) cell. The charging time constant, $\tau_{c}$, for the cell (plasma) membrane is given as:

$\tau_{c}=\left[\left\{\left(1+2 v_{\text {org }}\right) /\left(1-v_{\text {org }}\right)\right\} \rho_{1} / 2+\rho_{2}\right] C_{m} a$------ (12)

$V$ is the volume concentration of the spherical cells, $\rho_{1}$ is the resistivity of the suspending medium, $\rho_{2}$ the resistivity of the cytoplasm, $C_{m}$ the capacitance of the membrane per unit area, and $a$ is the cell diameter. The electric field in the membrane at the poles of the cell can be estimated from the condition that the current density at the interface of cytoplasm and membrane is continuous.

$\sigma_{M}=\sigma_{c}$----- (13)

The condition that the total voltage across the intraorganelle of the cell, $V_{n}$, is the sum of the voltage across the cytoplasm and twice that of across the membrane. Assuming the electric field along the axis of a cell with diameter $\mathrm{D}$, and membrane thickness, d, is constant in the cytoplasm and in the membrane, respectively, gives us:

$E_{c} D+2 E_{M} d=V_{n}$------ (14)

Combining the two equations allows us to calculate the electric field in the membrane, depending on the applied voltage,

$E_{M}=V_{c} /\left(\left(\sigma_{M} / \sigma_{c}\right) D+2 d\right)$.

Since the conductivity of the cytoplasm is generally on the order of $1 \mathrm{~S} / \mathrm{m}$, and the conductivity of the membrane is on the order of (10-5) $\mathrm{ms} / \mathrm{m}^{2}$, the first term in the denominator is small compared to the second. Consequently:

$E_{M} \approx V_{c} / 2 d$

This means that it is reasonable to assume that the entire voltage across a cell in this case is applied across the membranes (at the poles of the cell). This can lead to a strong thermal loading of the cell membranes at these positions. The energy density $(W)$ deposited in the membrane.

$W=\sigma_{M} \mathrm{E}_{\mathrm{M}}{ }^{2} \tau$-------- (17)

The time-dependent intraorganelle voltage $V_{n}(t)$ produces electro pores with a distribution of radii. Essentially, the Smoluchowski equation (SE) governs the growth and decay of pores and their evolution in radial-space. The continuum Smoluchowski theory yields the following equation for the pore density distribution function $n(r, t)$, with $r$ being the pore radius and $t$ the time variable [192].

$\partial n(r, t) / \partial t-\frac{\left\{D /\left(K_{B} T\right)\right\} \delta[n(r, t)(\delta E / \delta r)]}{\delta r}-\frac{D \delta^{2} n(r, t)}{\delta r^{2}}(r)-(18)$
Where $S(r)$ is the source (or pore formation) term, while D is a pore diffusion constant, and $\mathrm{E}(\mathrm{r})$ is the pore-formation energy. The pore formation " $\mathrm{S}$ " term depends on the transmembrane potential and has a strong exponential on its magnitude. The equation 17 and 18 shows that the pore energy is completely influenced by radius of nanopore which are generated into the intraorganelle.

\subsection{Simulation model bio chip}

\subsubsection{Design of bio chip}

Table 1 Design consideration of the micro chip in COMSOL software[37]

\begin{tabular}{|c|c|c|c|}
\hline Devices & Parameter & Value & Unit \\
\hline \multirow[t]{5}{*}{ Chip } & Length & 2300 & $\mu m$ \\
\hline & Height & 100 & $\mu m$ \\
\hline & Width & 900 & $\mu m$ \\
\hline & Inlet & 10 & $\mu m$ \\
\hline & Outlet & 10 & $\mu m$ \\
\hline \multirow{8}{*}{$\begin{array}{l}\text { Micro } \\
\text { Electrode }\end{array}$} & Length & 1000 & $\mu m$ \\
\hline & Width & 900 & $\mu m$ \\
\hline & Height & 100 & $\mu m$ \\
\hline & $\begin{array}{l}\text { Inter electrode } \\
\text { gap }\end{array}$ & & $\mu m$ \\
\hline & Central part & 50 & $\mu m$ \\
\hline & Medial part & 150 & $\mu m$ \\
\hline & Lateral part & 250 & $\mu m$ \\
\hline & Material & $\mathrm{Au}, \mathrm{Bi}$ & \\
\hline \multirow{3}{*}{$\begin{array}{l}\text { Micro } \\
\text { channel(Bi- } \\
\text { lateral) }\end{array}$} & Length & 2300 & $\mu m$ \\
\hline & Width & 250 & $\mu m$ \\
\hline & Height & 100 & $\mu m$ \\
\hline
\end{tabular}

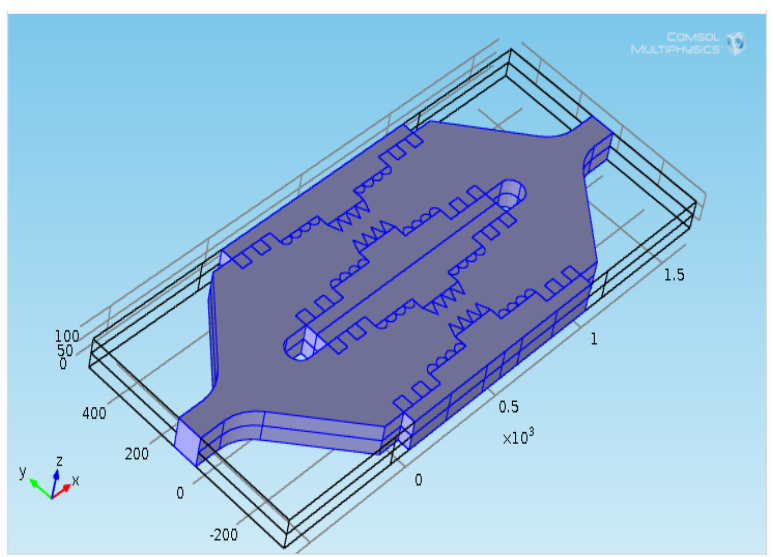

Fig 1: Design view of 3D micro device. 
It is reported that the effective nanoporation in intraorganelle membrane or nucleolus membrane need specific type of pulse, microfloudic chip and suspension media. It is reported that the optimum value of the applied pulse having the duration of 5 pico second, pulse intensity of 1 volts is suitable and effective intraorganelle nanoporation of osteoblast cell. it also exposed that intraorganelle nanoporation can easily obtained if the shape of the electrode is triangular or saw tooth which are made by gold bismuth alloy in specified micro channel having the height and width of micro -fluidic channel should be moderate and same \& it would be taken as 200 micrometer for successful and effective intraorganelle nanoporation. The resistances of micro channel should be as high as possible for above purpose. With the help of above discussion the author optimised the system and design a simulating micro fluidic chip on the bases of following specification with in which the osteoblast cell is placed ,to explore the numerical and analytical characterization of intraorganelle nanoporation. The simulation tool is composed of a $100 \mu \mathrm{m}$ thick SU8 micro fluidic channel including thick bi metallic electrode (Bi and $\mathrm{Au}$ ) electrodes with a typical thickness of $50 \mu \mathrm{m}$, in which cells suspended in a biological medium are injected. Bimetallic is chosen as material for the electrodes because of its excellent electrical properties and bio compatibility. The biochip is designed in such a way that the pulsed electric field is absorbed and dissipated mainly in the biological medium placed between the electrodes within which cells to be treated are flowed.

\subsubsection{Characterization of Micro chip}

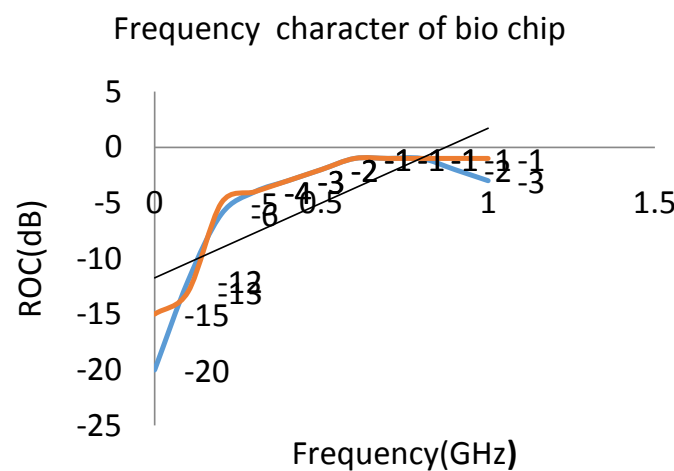

Fig 2: Frequency domain characterization of biochip with measurements of reflection coefficient.

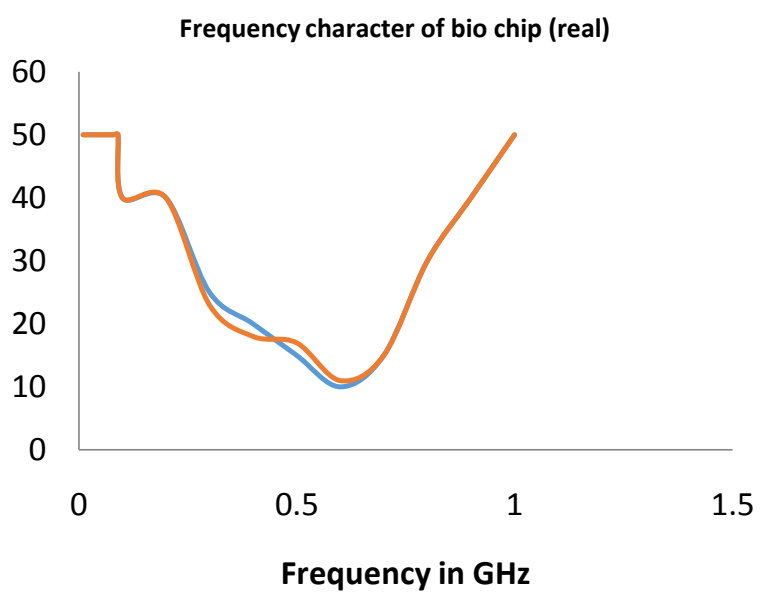

Fig 3: Frequency domain characterization of biochip, measurements of impedances.
Rice time $0.5 \mathrm{ps}$

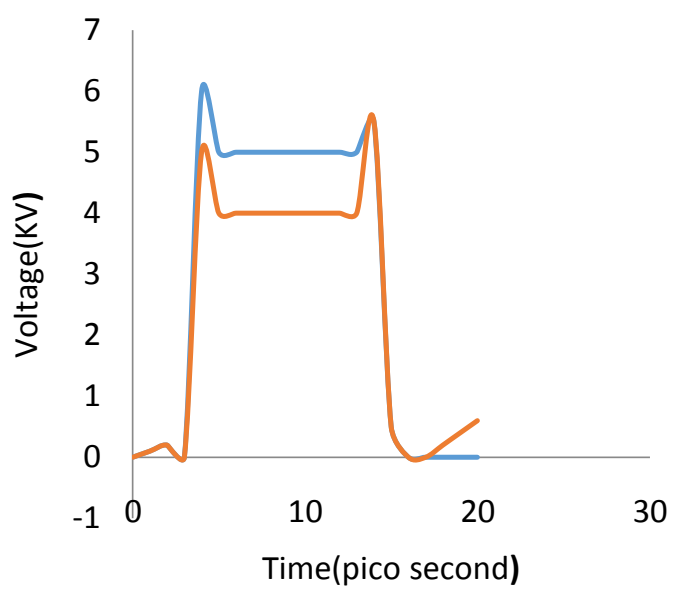

Fig 4: Time domain characterization of biochip, Measurements of voltage applied and generated within the bio chip. (Rice time $-0.5 \mathrm{ps}$ ).

Rice time (1.5 ps)

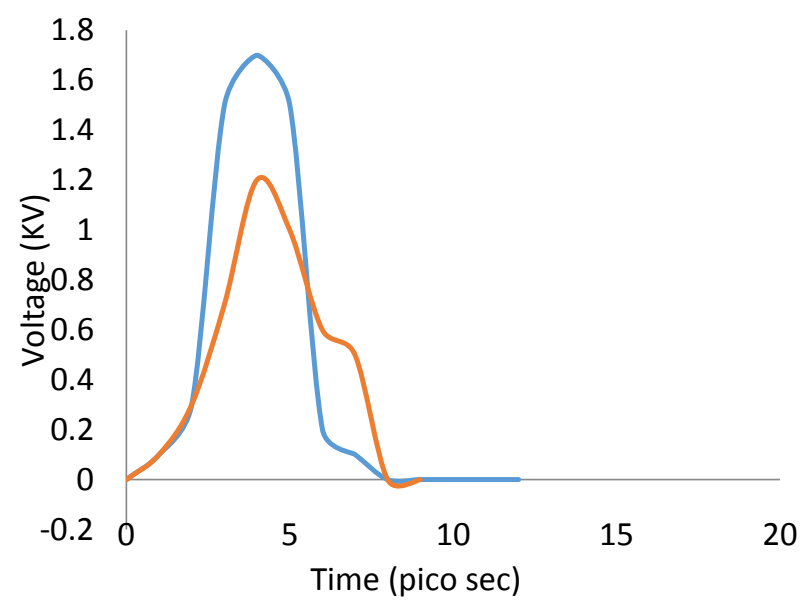

Fig 5: Time domain characterization of biochip, Measurements of voltage applied and generated within the bio chip. (Rice time -1.5 ps)

The biochip is validated by its frequency and time domain analysis. The electromagnetic property is measure by LCR meter along with dielectric probe and relative permittivity and reflection co efficient are measured by vector network analyser. The impedance is calculated from the reflection coefficient. The figure 18 shows the reflection coefficient and fig 2 explores the real part of impedances of bio chip. The measured results present a very good level of consistency. The good impedance matching is obtain up to $100 \mathrm{MHz}$.As shown in fig 3 at low frequency the biochip is purely resistive but in high frequency the impedance of bio chip is reduced. In figure 4and 5 the voltage is measured ,i.e. generated and applied pulses are present and it explores the good level of consistency .The rice time is slightly modified by the biochip and make it reliable for intraorganelle nanoporation 


\section{CHARACTERIZATION OF OSTEO NANOPORATION}

\subsection{Life Time of Nanopore}

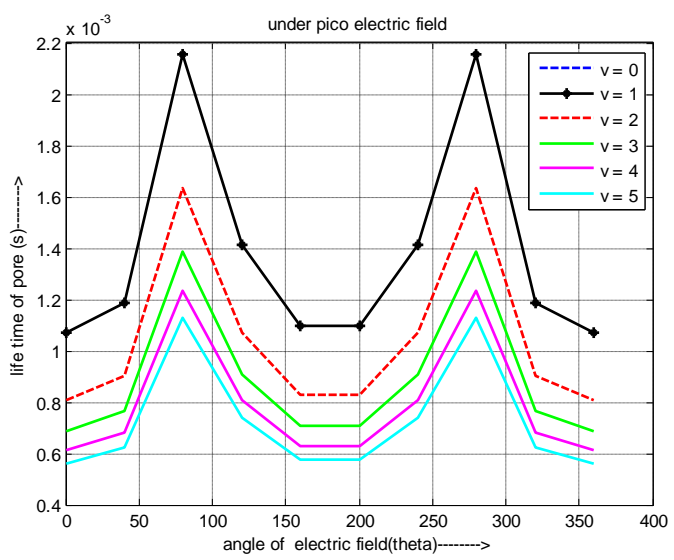

Fig6: Variation of life time of pore with respect to azimuthal angle in different pulse intensity

The Fig 6 explores the variation of intra organelle pore life time along with pole position of applied electric field for intraorganelle It clears that the life time of all the pores are not same. it is nonuniformly distributed for all the pores.It is also shown that pore life time is gradually increase as the angle of applied electric field is increase \& maximum is obtain at an angle of $\theta=90$ and $\theta=270$ which is independent of pulse, electrode, micro channel and suspension media specification due to different molecular structure of intraorganelle membranes.

\subsection{Pore radius}

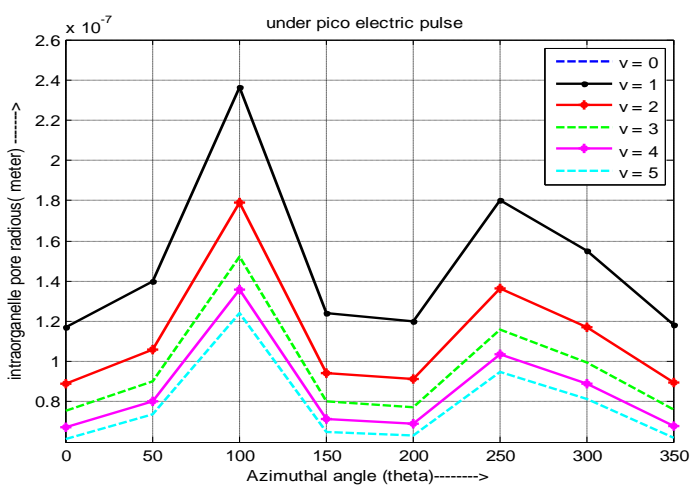

Fig7: Variation of intraorganelle pore radius with respect to azimuthal angle in different pulse intensity

The Fig 7 shows the variation of intra organelle pore radius along with pole position of applied electric field in different pulse intensity uder the influences of pico electric field. It clears that the radius of all the pores are not same it is sinusoidal distributed over the rmembrane.. As the nanopores are created, intra organelle potential increases and the biggest nanopores move just opposite to the equator (E). It is also shown that pore radius is gradually increase as the angle of applied electric field is increase \& maximum pore radius is obtain at an angle of $\theta=100$ which is independent of pulse, electrode, micro channel and suspension media specification due to the higher elasticity of layers.

\subsection{Ion Selectivity of nanopores}

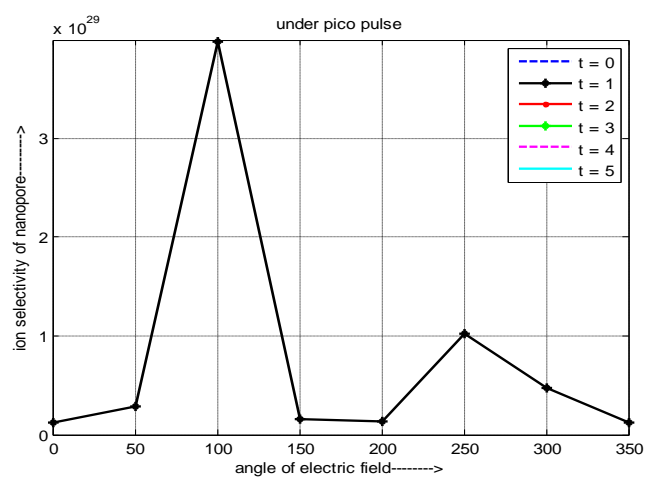

Fig8: Variation of ion selectivity with respect to azimuthal angle in different pulse intensity

The Fig 8 shows the variation of intra organelle ion uptake along with pole position of applied electric field for intraorganelle It is observed that the amount of ion which is uptake by the intra organelle is only occurred at pico scale pulse and its value is not same throughout the whole surface of the layer of the nucleus.. The maximum ion uptake occurs at pole $(\theta=100 \& 250)$ where the surface tension is minimum and it implies that such part of membrane will inherently have an inverse Kelvin vapor pressure effect, that resulting in increased water condensation.

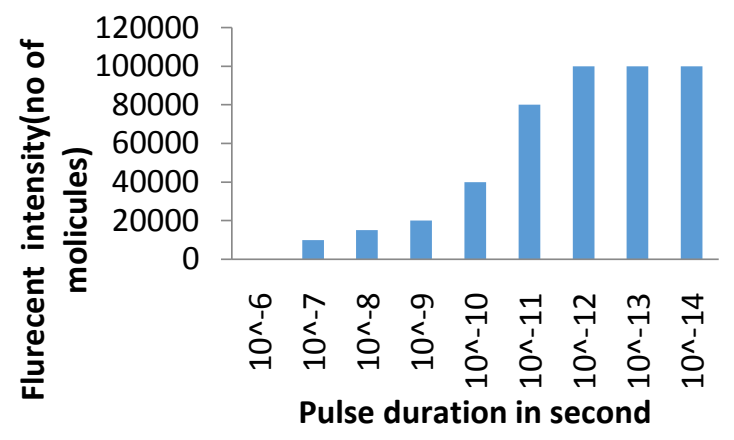

Fig 9: Nanoporation of a typical osteoblast cell undergoing PI uptake.

\section{osteo nanoporation}

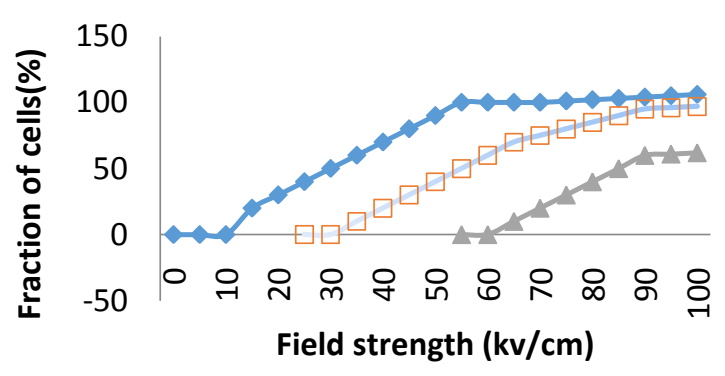

Fig. 10. Dependences of the fraction of osteoblast cell , which were nanoporated by a single square-wave electric pulse. 


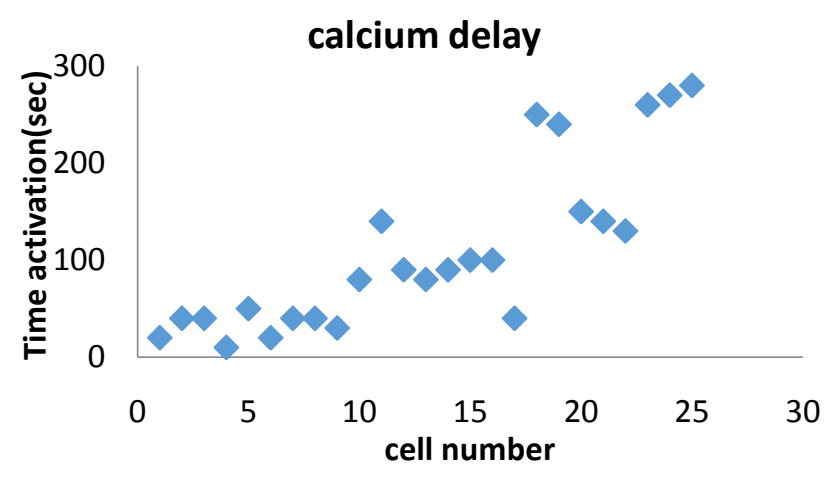

Fig 11. The delay between beginning of stimulation and maximum calcium increase in every cell

\section{FLURESCENT INCREASE}

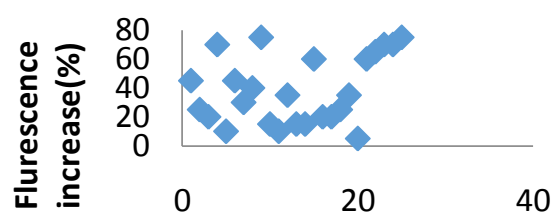

cell number

Fig 12: the ratio increase in every cell.

From this observation it is exposed that there is no effect of micro pulse but pico pulse penetrate the on intraorganelle.It supports the numerical and analytical result explore in this research.In this research papers, the fraction of the osteoblast cell that were nanoelectroporated was determined from the experiment as explained above.It should be noted that the right information of the cell nanoporation is rather complex. Fig. 9 shows the dependences of the fraction $(F \mathrm{p})$ of electroporated cells versus the amplitude of the single squarewave electric field pulse, determined for pulses whose duration varied from 50 to $100 \mathrm{ps}$. The amplitude of the electric pulse was changed from 0 to $100 \mathrm{kV} / \mathrm{cm}$. From Fig. 9, an increase of the pulse amplitude or duration increases the fraction of the cells being electroporated. For the 100-ps duration pulse, pores permeable to sodium ions appeared in the cell plasma membrane at $10-\mathrm{kV} / \mathrm{cm}$ electric field strength. The field required to electroporate $50 \%$ of the cells in this case was $\sim 20 \mathrm{kV} / \mathrm{cm}$. When the pulse duration was decreased to $75 \mathrm{ps}$, the strength of the electric field required to electroporate $50 \%$ of the cells increased up to $65 \mathrm{kV} / \mathrm{cm}$. In the 50-ps duration pulse with amplitude of $110 \mathrm{kV} / \mathrm{cm}$, only about $20 \%$ of osteoblast like cells was nanoporated. To date, there has been a lack of studies in which the threshold of the intraorganelle nanoelectroporation was determined for pulses $=100$ ps. However, the fraction of the cells that were electroporated was not estimated.It is observed thst when this process for increasing the permeability of the intraorganelle osteoblast like cell membrane (because of picosecond pulse) to the smallest ions $(\mathrm{Na}+\mathrm{K}+$, and $\mathrm{Rb}+)$ is used, this makes it possible to detect with sufficient accuracy the threshold of the field of poration and allows one to determine the dependence of this field on the pulse duration. It also has to be noted that the electric field strength required to porate the cell plasma membrane using picosecond pulses increases more abruptly with the decrease of the pulse duration.

Since not all cells responded at the same time with a calcium increase (Fig 11 and 12,)calcium responses were analyzed in steps of 50 seconds. Thus it was identified the number of cells that reached their peak value in the first 50 seconds, in 100 seconds, etc. It was compared the Peak value in cell number percentage This mean that was compared the percentage of cells that reached their maximum calcium concentration in steps of 50 seconds. It was evident that most cells (50\%) had their maximal calcium increase in the first 50 seconds and then cell number percentage was decreasing as time went by. Thus a shorter period of stimulation may have a greater influence intracellular rearrangement.This concept is key resources of our idea and we explore concept for nanopore formation over the intraorganelle of osteoblast like cell.

\section{APPLICATION \\ 4.1.Intraorganelle nanoporation for Drug and Gene Delivery}

Practically,Intraorganelle nanoporation is used to deliver drugs or other molecules opposed by intraorganelle membranes.Intraorganelle nanoporation has been shown to be safe and effective in humans to overcome the With potential problems for DNA delivery by viruses. It was also observed that only minor histological changes occurred in osteo intraorganelle during nanoporation with neither little or no changes in gene profiles nor in DNA stability.One practical medical application for Intraorganelle nanoporation is to permeabilize tumor cell membranes for the delivery of poorly permeable drugs, such as electrochemotherapy and ultra sound in different developed countries.

\subsection{Nanoporation for cancer treatment}

Electro-gene therapy is widely used for cancer treatments. Initial thoughts were to use EGT for replacing defective genes. However, a major focus has been on several basic strategies for cancer therapy, including immune potentiation, suicide gene therapy, restoration of tumor suppressor genes, inhibition of oncogenes, anti-angiogenic gene therapy, delivering genes encoding toxins, or siRNAs to knockdown proteins important for survival and growth.In addition, 2 of 19 patients with non-electroporated distant lesions and no other systemic therapy showed complete regression of all metastases. Other studies found that high antibody titers after Intraorganelle nanoporation delivery of a plasmid encoding .Cell survival and DNA delivery during Intraorganelle nanoporation of course EGT requires that cells survive Intraorganelle nanoporation treatment, so they can express the gene of interest. Finally, there is a threshold that completely compromises cell viability, such that cells are irreversibly permeabilized .It is now clinically used for liver cancer.

\subsection{Intracellular effects of nanoporation}

Unlike conventional Intraorganelle nanoporation pulses, nanosecond pulses are hypothesized to affect intracellular membranes. Therefore, a major focus has been on nsPEF effects on the endoplasmic reticulum, other calcium stores, mitochondria. The initial concept of nsPEF effects was that 
plasma membranes were not affected or less affected. In experiments with cells in vitro, calcium did not escape from intracellular vesicles into the cytoplasm and propidium iodide (PI) did not initially enter cells, suggesting that plasma membranes remained intact. Later it became clear that breaches in plasma membranes were too small to allow escape or admission of molecules with sizes larger than about a nanometer, slighter smaller than calcium and PI.When nsPEFs were shown to eliminate cancer cells, evidence was interpreted as markers for apoptosis and necrosis. Such mechanisms could generally result from effects on plasma membranes, intracellular membranes, or both.

\subsection{Nanoporation used in intrinsic cell death.}

It was hypothesized that nsPEF-induced apoptosis resulted from bsupra-Intraorganelle nanoporation . Again, this could be due to extrinsic effects on plasma membranes, or intrinsic effects on endoplasmic reticulum, mitochondria and/or nuclei/DNA. Lysosomes could also be affecting, but have not been analyzed.

\subsection{Nanoporation used in extrinsic cell death.}

When these clones were treated with ten $60 \mathrm{~ns}$ pulses with increasing electric fields $(0-60 \mathrm{kV} / \mathrm{cm})$, there were no differences in electric field effects on cell viability.However,it did show that like other apoptosis stimuli, nsPEFs activate similarcaspase-independent cell death mechanisms in the absence of caspase activities.To more specifically test for nsPEF-activated extrinsic apoptosis,

\subsection{Sub cellular Targets and Cell Death Pathways Activated By Nanoporation}

Our present understanding of possible sub cellular targets and cell death mechanisms in response to nsPEFs, and summarizes the finding presented above. Based on the evidence presented above and elsewhere, mitochondria.

\section{CONCLUSION}

The numerical and analytical model of intra organelle nano poration of osteoblast cell placed in a 3D hybrid micro biochip under the influences of pico pulse has been reported. This study encourage the specific micro chip multiple dimension microfloudic channel with irregular bi metallic ( $\mathrm{Bi}$ and $\mathrm{Au}$ ) side wall electrodes that are designed to deliver a maximum of energy to the biological medium containing multilayer osteoblast cell. The reported nanoporative device aided by a quantitative understanding of the interactions between cells and an external electric field to provide more advantageous conditions than conventional systems with small voltages and power consumptions, continuous flow, small sample volume, and negligible heating.

Intraorganele nanoporation is a promising technique for targeted drug delivery with various applications in cancer therapy. The initial studies on the treatment of superficial and deep seated tumours with intraorganele nanoporation showed significant outcomes. To improve the intraorganele nanoporation as a new modality in cancer treatment, different studies should be performed to determine the mechanisms of action of the technique. Conducting in-vivo and in-vitro experimental, as well as exact modelling studies can shed light on the different aspects of mechanisms of action of this technique. In summary, the present data provide evidence that psPEF introduce the ions with specified microchip possibly through the nucleus mediated pathway. The use of picoseconds pulses not only allows entering a new field of field-cell interactions, but it may open the door to a range of non-invasive therapeutic applications. Further studies are needed to elucidate the osteoblast cell responses to psPEF in detail within the micro fluidic environment above $\mathrm{GHz}$ frequency range.

\section{REFERENCES}

[1] Tsong, T.Y., 1991. Electroporation of cell-membranes. Biophysical Journal 60(2), 297-306.

[2] Marty, M., Sersa, G., Garbay, J.R., Gehl, J., Collins, C.G., Snoj, M., Billard, V., Geertsen, P.F., Larkin, J.O.,Miklavcic, D., Pavlovic, I., Paulin-Kosir, S.M., Cemazar, M., Morsli, N., Rudolf, Z., Robert, C., O'Sullivan, G.C.,Mir, L.M., 2006. Electrochemotherapy An easy, highly effective and safe treatment of cutaneous and subcutaneousmetastases: Results of ESOPE (European Standard Operating Procedures of Electrochemotherapy) study. Ejc Supplements 4(11), 313.

[3] Mir, L.M., 2006. Electrochemotherapy - An easy, highly effective and safe treatment of cutaneous and subcutaneous metastases: Results of ESOPE (European Standard Operating Procedures of Electrochemotherapy) study. Ejc Supplements 4(11), 3-13.

[4] Mir, L.M., Glass, L.F., Sersa, G., Teissie, J., Domenge, C., Miklavcic, D., Jaroszeski, M.J., Orlowski, S., Reintgen, D.S., Rudolf, Z., Belehradek, M., Gilbert, R., Rols, M.P., Belehradek, J., Bachaud, J.M., DeConti, R., Stabuc, B., Cemazar, M., Coninx, P., Heller, R., 1998. Effective treatment of cutaneous and subcutaneous malignant tumours by electrochemotherapy. British Journal of Cancer 77(12), 2336-2342.

[5] Mir, L.M., Gehl, J., Sersa, G., Collins, C.G., Garbay, J.R., Billard, V., Geertsen, P., Rudolf, Z., O'Sullivan, G.C.,Marty, M., 2006. Standard operating procedures of the electrochemotherapy: Instructions for the use of bleomycin or cisplatin administered either systemically or locally and electric pulses delivered by the CliniporatorTM by means of invasive or non-invasive electrodes. Eur J Cancer S4, 14-25.

[6] Labanauskiene, J., Gehl, J., Didziapetriene, J., 2007. Evaluation of cytotoxic effect of photodynamic therapy in combination with electroporation in vitro. Bioelectrochemistry 70(1), 78-82

[7] Gothelf, A., Gehl, J., 2010. Gene Electrotransfer to Skin; Review of Existing Literature and Clinical Perspectives.Current Gene Therapy 10(4), 287-299.

[8] Andre, F., Mir, L.M., 2004. DNA electrotransfer: its principles and an updated review of its therapeutic applications. Gene Therapy 11, S33-S42.

[9] Buescher, E.S., Smith, R.R., Schoenbach, K.H., 2004. Submicrosecond intense pulsed electric field effects on intracellular free calcium: mechanisms and effects. Plasma Science, IEEE Transactions on 32(4), 1563-1572.

[10] Vernier, P.T., Sun, Y.H., Gundersen, M.A., 2006. Nanoelectropulse-driven membrane perturbation and small molecule permeabilization. Bmc Cell Biology 7.

[11] Vernier, P.T., Thu, M.M.S., Marcu, L., Craft, C.M., Gundersen, M.A., 2004. Nanosecond electroperturbation 
-Mammalian cell sensitivity and bacterial spore resistance. Plasma Science, IEEE Transactions on 32(4), 1620-1625.

[12] Beebe, S.J., Schoenbach, K.H., 2005. Nanosecond pulsed electric fields: A new stimulus to activate intracellular signaling. Journal of Biomedicine and Biotechnology(4), 297-300.

[13] Beebe, S.J., White, J., Blackmore, P.F., Deng, Y.P., Somers, K., Schoenbach, K.H., 2003. Diverse effects ofnanosecond pulsed electric fields on cells and tissues. DNA and Cell Biology 22(12), 785-796.

[14] E. Neumann, A.E. Sowers, C.A. Jordan (Eds.), Electroporation and Electrofusion in Cell Biology, in, Plenum, New York, 1989.

[15] U. Zimmermann, G.A. Neil, in: Electromanipulation of Cells, CRC Press, BocaRaton, 1996.

[16] A.G. Pakhomov, D. Miklavcic, M.S. Markov (Eds.), Advanced ElectroporationTechniques in Biology in Medicine, CRC Press, Boca Raton, 2010, p. 528.

[17] Irreversible electroporation, in: B. Rubinsky (Ed.), Series in Biomedical Engineering,Springer-Verlag, Berlin Heidelberg, 2010.

[18] K.H. Schoenbach, S.J. Beebe, E.S. Buescher, Intracellular effect of ultrashort electrical

pulses, Bioelectromagnetics 22 (2001) 440-448.

[19] K.H. Schoenbach, Bioelectric effect of intense nanosecond pulses, in: A.G.Pakhomov, D. Miklavcic, M.S. Markov (Eds.), Advanced Electroporation Techniquesin Biology in Medicine, CRC Press, Boca Raton, 2010, pp. 19-50.

[20] T.R. Gowrishankar, J.C. Weaver, Electrical behavior and pore accumulation in amulticellular model for conventional and supra-electroporation, Biochem.

Biophys. Res. Commun. 349 (2006) 643-653.

[21] T. Kotnik, D. Miklavcic, Theoretical evaluation of voltage inducement on internalmembranes of biological cells exposed to electric fields, Biophys. J. 90 (2006)

480-491.

[22] G.L. Craviso, S. Choe, P. Chatterjee, I. Chatterjee, P.T. Vernier, Nanosecond electric

Pluses: a novel stimulus for triggering $\mathrm{Ca} 2+$ influx into chromaffin cells viavoltage-gated $\mathrm{Ca} 2+$ channels, Cell. Mol. Neurobiol. 30 (2010) 1259-1265.

[23] P.T. Vernier, Y. Sun, M.A. Gundersen, Nanoelectropulse-driven membrane perturbation

and small molecule permeabilization, BMC Cell Biol. 7 (2006) 37.

[24] A.G. Pakhomov, J.F. Kolb, J.A. White, R.P. Joshi, S. Xiao, K.H. Schoenbach,Long-lasting plasma membrane permeabilization in mammalian cells by nanosecondpulsed electric field (nsPEF), Bioelectromagnetics 28 (2007) 655-663.

[25] A.G. Pakhomov, R. Shevin, J.A. White, J.F. Kolb, O.N. Pakhomova, R.P. Joshi, K.H.Schoenbach, Membrane permeabilization and cell damage by ultrashort electricfield shocks, Arch. Biochem. Biophys. 465 (2007) 109-118.

[26] W. Frey, J.A. White, R.O. Price, P.F. Blackmore, R.P. Joshi, R. Nuccitelli, S.J. Beebe,K.H. Schoenbach, J.F. Kolb, Plasma membrane voltage changes during nanosecondpulsed electric field exposure, Biophys. J. 90 (2006) 3608-3615.

[27] Brighton, C. T., and S. R. Pollack. 1985. Treatment of recalcitrant non-union with acapacitively coupled electric fields. J. Bone Jt. Surg. 67A:577-585.

[28] Scott, G. and King J. B. 1994. A prospective doubleblind trial of electrical capacitivecoupling in the treatment of nonunion of long bones. J. Bone Jt. Surg. 76A:820-826.

[29]. Hall, B. K.1990. BONE, Volume VII. The Telford Press, Cadwell, New Jersey, USA.

[30]. McLeod, K J., and C. T. Rubin. 1992. The effect of low frequency electric fields onosteogenesis. Journal of bone and Joint surgery, 74A:920-929.

[31]. Ferrier, J., S.M. Ross and J. Aubin. 1986. Osteoclasts and osteoblasts migrate inopposite directions in response to a constant electrical field. J. Cell. Physiol. 129:283-

288.

[32].Brighton C. T., E. Okereke and R. Pollack. 1992. In vitro bone-Cell response to acapacitively coupled electric field. Clin. Orthop. 285:255-262.

[33]. McLeod, K.J., B. J. Donahoe, P.E. Levin, M. A. Fontaine and C. T. Rubin. 1993.Electric fields modulate bone cell function in a density-dependent manner. J BoneMiner Res 8:977-984.

[34]. Hartig, M., U. Joos and H-P Wiesmann. 2000. Capacitively coupled electric fieldsaccelerate proliferation of osteoblasts-like primary cells and increase boneextracellular matrix formation in vitro. Eur Biophys J 29:499-506.

[35] Sher LD, Kresch E, Schwan HP: On the possibility of nonthermal biological effects of pulsed electromagnetic radiation. Biophys J 1970, 10(10):970-979.

[36] Schoenbach KH, Joshi RP, Kolb JF, Chen NY, Stacey M, Blackmore PF, Buescher ES, Beebe SJ: Ultrashort electrical pulses open a new gateway into biological cells. Proceedings of the IEEE 2004,92(7):1122-1137.

[37] S.Sarkar.R.Mahapatra, M.K.Ghosh,"Study of Intra Organelle Nanoporation of Multilayer Dense Osteoblast Cell" International Journal of Computer Applications, Vol. 87(1), pp.17-22,(2014). 